\title{
Inhomogeneous diffusion model for recent data on high-energy cosmic rays
}

\author{
Nicola Tomassetti* \\ LPSC, Université Grenoble-Alpes, CNRS/IN2P3, F-38026 Grenoble, France; email: \\ nicola.tomassetti@lpsc.in2p3.ch \\ E-mail: nicola.tomassettialpsc.in2p3.fr
}

The AMS Collaboration has recently released precision data on cosmic ray (CR) leptons and protons at high energies. Interesting progresses have also been made on the measurement of CR nuclei, such as the boron-to-carbon ratio or the lithium spectrum, up to TeV/nucleon energies. In order to provide a description these data, I consider a diffusion model of CR propagation which allows for latitudinal variations of the CR diffusion properties in the Galactic halo. I discuss the role of high-precision data on light CR nuclei in resolutely testing this model and the key propagation parameters.

The 34th International Cosmic Ray Conference,

30 July- 6 August, 2015

The Hague, The Netherlands

${ }^{*}$ Speaker. 


\section{Introduction}

The observed properties of Galactic cosmic rays (CRs) are believed to arise from a combination of two basic plasma astrophysics phenomena: diffusive shock acceleration mechanisms, occurring in supernova remnants, and diffusive trasport processes off magnetic turbulence $[1,2,3]$. The paradigm of CR transport as a diffusion process off magnetic irregularities has been established for decades. It may explain the observed high-degree of isotropy on the $\mathrm{CR}$ arrival directions or the abundance of the secondary Li-Be-B elements that are generated from CR collisions with the matter. The so-called standard models based on this understanding employ several simplifying assumptions. In practical implementations, the CRs are assumed confined inside a cylindrical halo, that encompasses the Galactic plane, after being released by supernova remnants with power-law acceleration spectra $Q \propto E^{-v}$. The diffusion coefficient is assumed to be $K(E) \propto E^{\delta}$, spatially homogeneous and isotropic in the whole halo. The combined effects of acceleration and transport leads to power-law spectra $\phi(E) \sim Q / K \propto E^{-\delta-v}$ for primary components (protons, He, C, O, $\mathrm{Fe}$ ) and a dependence $\sim E^{-\delta}$ for secondary/primary ratios. The data on primary spectra and on the $\mathrm{B} / \mathrm{C}$ ratio constrain the parameters $\delta \approx 0.3-0.7$ and $v \approx 2-2.4$ [4].

Recent experiments such as AMS PAMELA or CREAM have found exciting features in the $\mathrm{CR}$ spectrum that may suggest the need of a revision of some standard model assumptions. The rise of the positron fraction $\mathrm{e}^{+} /\left(\mathrm{e}^{-}+\mathrm{e}^{+}\right)$at $\sim 10-300 \mathrm{GeV}$ of energy $[5,6]$, in contrast to the standard expectations, may suggest the presence of dark-matter or astrophysical sources of high-energy $e^{ \pm}$, as well as the need to reassess the secondary $e^{ \pm}$secondary production $[7,8,9,10,11]$. The spectral hardening observed in CR protons and nuclei at $E \sim 300 \mathrm{GeV} /$ nucleon [12, 13, 14, 15] may be a signature of new astrophysical phenomena, occurring either in acceleration or in propagation, that are not accounted by the standard models $[16,17,18,19,20]$. Revisiting CR propagation is particular suggestive given its connection with the so-called "anisotropy problem". The data on CR anisotropy amplitude prefers a rather shallow dependence of the high-energy diffusivity in comparison with that inferred from the $B / C$ ratio [34]. A change on diffusion is also hinted by a high-energy flattening on the secondary to primary ratios. New $\bar{p} / p$ data presented by AMS have generated widespread interest in connection with for the dark matter searches or with the astrophysical diagnostics of CR propagation [21, 22, 23].

In the following, we present a numerical calculation of CR spectra under a two halo scenario of inhomogeneous diffusive transport. In this scenario, we assume that the diffusion is shallower when CRs propagate close to the Galactic disk, based on the idea that the two regions are characterized by a different of magnetic turbulence regimes. This scenario represents the simplest but physically consistent generalization of the standard models that generally employ a homogeneous diffusivity in the whole propagation region. In standard models, even when a spatial dependence of $\mathrm{CR}$ diffusion is considered, the energy dependence of the diffusion coefficient is always assumed to be "universal", i.e., standard models assume a unique diffusion regime in the whole Galaxy. On the contrary, we believe that the different types of turbulence observed in the Galaxy lead to different energy dependence of CR diffusion. By the mathematical point of view, this suggestion is formalized by a spatial dependent diffusion coefficient which is not separable into energy and space coordinates. As we will show, the two-halo scenario of CR diffusion is supported by several CR data, with important consequences for the physics observables that are being investigated by 

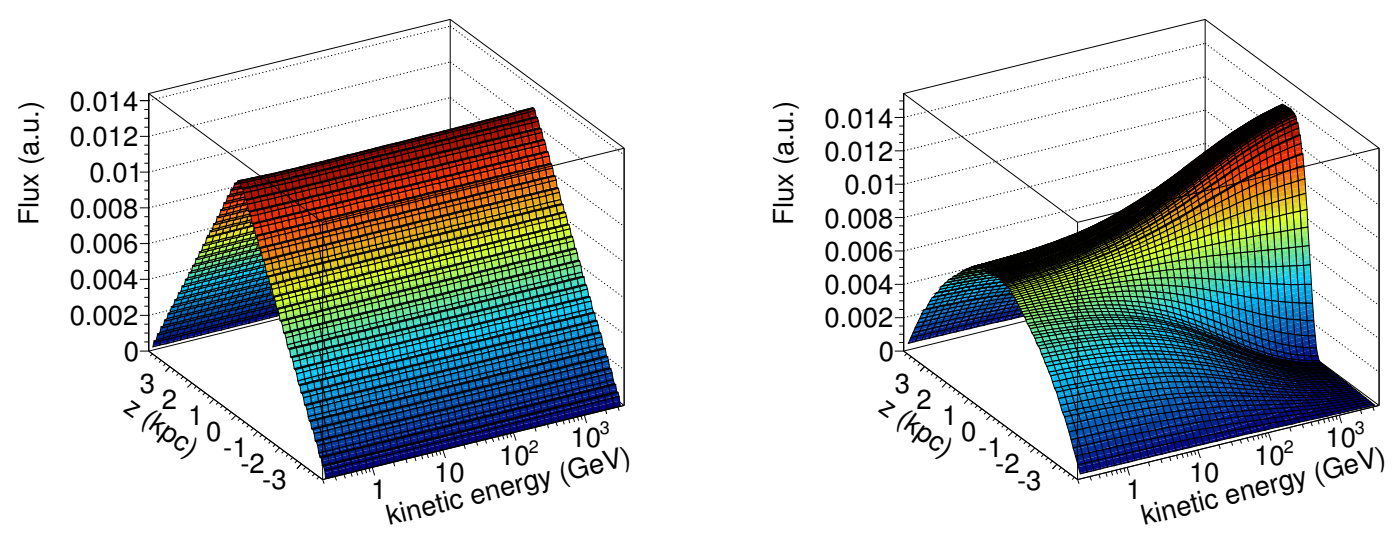

Figure 1: CR flux density for primary protons as function of energy and the spatial $z$-coordinate for the standard diffusion model (left) and from the inhomogeneous model (right).

AMS. In particular, we will comment its implications for dark matter searches via antimatter.

\section{The model}

We define a phenomenological scenario with two propagation regions, i.e., two halos, characterized by different energy dependence of the diffusion coefficient. The inner halo represents a thin region, surrounding the disk by a few hundred pc, where the turbulence of the medium is influenced by supernova explosions. The outer halo represents a wider region, extended up to $z=L$, where the turbulence is presumably generated by CRs themselves, given that supernova explosions do not occurs away from the disk. To numerically set the model, we employ the DRAGON package [24], which solves the diffusion equation for a given distribution of sources, providing equilibrium solutions for the interstellar spectra of nuclei and leptons. We introduced a modification on the finite-differencing scheme and on the spatial grid in order to have a latitudinal dependence for the parameter $\delta \equiv \delta(z)$, The implementation is close to the work in [25]. The vertical extent of the full propagation region is $L=4 \mathrm{kpc}$. The two halos are sized $l_{i}=\xi L$ and $l_{o}=(1-\xi) L$, with $0<\xi<1$. The diffusion coefficient is $K(R, z)=\beta K_{0}\left(R / R_{0}\right)^{\delta(z)}$. With this functional form, the diffusion coefficient $K$ is non-separable into energy and space coordinates. Its spatial dependence is defined by $\delta=\delta_{i}$ in the inner halo $(|z|<\xi L)$ and $\delta=\delta_{o} \equiv \delta_{i}+\Delta$ in the outer halo $\left(\xi_{L}<|z|<L\right)$. The two $\delta_{i / o}$ values are connected smoothly in order to ensure the continuity of $K(z)$ across the two zones. From the consideration we made, one has $\delta_{i}<\delta_{o}$, i.e., $\Delta>0$. In the following, we adopt the parameters $\delta=0.15, \Delta=0.5$, and $\xi=0.14$. The source spectra are of the type $Q(R)=\beta\left(R / R_{0}\right)^{-v}$ with index $v$ tuned to match the data. In this implementation, the nuclear physics inputs such as fragmentation, decays, energy losses are from Galprop code [4]. We also set up a standard model of Kraichnan-type diffusion, with $\delta \equiv 0.5$ everywhere in the whole halo, The solar modulation is described under the force-field approximation [26]. 

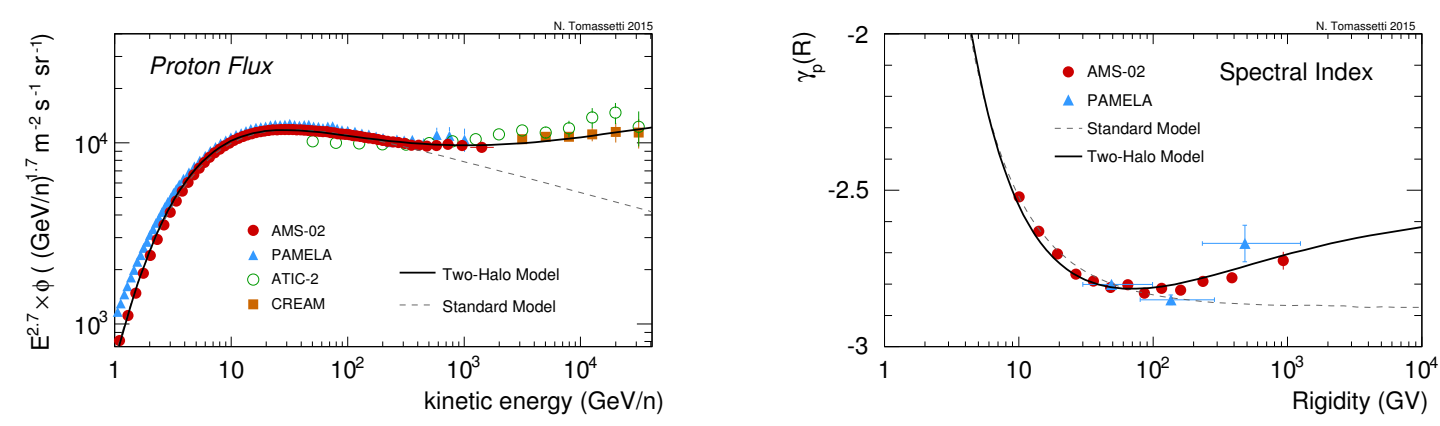

Figure 2: Left: Proton energy spectrum multiplied by $E^{2.7}$. Right: rigidity dependence of the proton spectral index. The model calculations are shown in comparison with the data $[15,12,13,14]$. The modulation potential is $\Phi=550 \mathrm{MV}$.

\section{Predictions for Primary Cosmic-Ray Hadrons}

The flux density as function of energy and of the spatial $z$-coordinate is shown in Fig. 1 for the proton spectrum. Other species have similar shapes. The particular shape of the two-halo model reflect the interplay of the $\mathrm{CR}$ diffusion into the two propagation halos. For the equilibrium fluxes expected at Earth, this interplay produces a remarkable departure from the standard power-law expectations. The proton spectrum is shown in Fig. 2, for the two models, in comparison with the data recently reported by AMS [15]. The AMS collaboration has now precisely measured the detailed variations of the proton and $\mathrm{He}$ fluxes at $\mathrm{GeV}-\mathrm{TeV}$ energies [15]. These data show a significantly smoother spectral hardening in comparison to that previously reported by PAMELA. In the figure we also compare the differential spectral index as function of rigidity, $\gamma(R)=d[\log (\phi)] / d[\log (R)]$, which is found to progressively harden at energy above $\sim 100 \mathrm{GeV}$ [15]. The results of this calculation nicely confirm the results previously obtained in our analytical derivations [17].

\section{Predictions for Secondary Cosmic-Ray Nuclei}

Secondary nuclei like boron, have steeper spectra due to diffusion. In our model, they also experience a stronger change of slope, due to the hardening factor of their progenitor nuclei and their subsequent diffusion in the two propagation zones. The $\mathrm{B} / \mathrm{C}$ ratio is therefore expected to progressively flatten at high-energy, with an asymptotic multi-TeV dependence $\sim E^{-\delta_{i}}$ which is determined by the inner halo diffusion properties. At low energy, our two-halo model remarkably recover the standard model behavior $\sim E^{-0.5}$. The existing $\mathrm{B} / \mathrm{C}$ data support this high-energy flattening. The $\mathrm{B} / \mathrm{C}$ ratio is currently being precisely measured by $\mathrm{AMS}$ in the $\mathrm{GeV}-\mathrm{TeV}$ energy region. In the figure we also shown the Lithium spectrum. Lithium is produced by secondary reactions of $\mathrm{C}-\mathrm{N}-\mathrm{O}$ nuclei with the ISM, like boron, but also from tertiary production reactions such as $\mathrm{B} \rightarrow \mathrm{Li}$ or $\mathrm{Be} \rightarrow \mathrm{Li}$ which steepen its low-energy spectrum. Though the existing measurements are affected by very large errors, the Li flux is currently being measured by AMS 1 to 1000 $\mathrm{GeV} /$ nucleon energies to a percent precision. Measurements of $\mathrm{B} / \mathrm{C}$ ratio and $\mathrm{Li}$ spectrum will allow to resolutely test our scenario. 

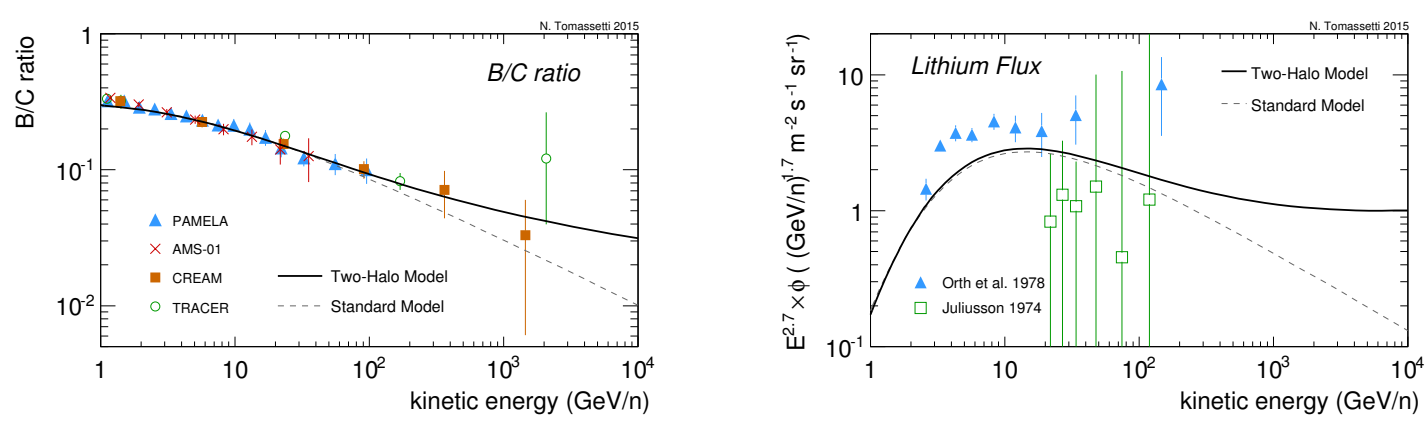

Figure 3: $\mathrm{B} / \mathrm{C}$ ratio and $\mathrm{Li}$ spectrum. The $\mathrm{Li}$ spectrum is multiplied by $E^{2.7}$. The model calculations are shown in comparison with the data $[27,28,29,14,30,32,33]$. The modulation potential is $\Phi=350 \mathrm{MV}$.

\section{Predictions for Secondary Cosmic-Ray Antimatter}

The sharp rise of the positron fraction $\mathrm{e}^{+} /\left(\mathrm{e}^{-}+\mathrm{e}^{+}\right)$at $\sim 10-300 \mathrm{GeV}$ of energy $[5,6]$, in contrast to the standard expectations $E^{-\delta}$, may suggest the presence of extra sources of high-energy $e^{ \pm}$, including dark matter particle annihilation/decay or nearby astrophysical sources as well as the need to reassess the secondary $e^{ \pm}$production from CR collisions with the gas $[7,8,9,10,11]$. Recent AMS data have triggered several speculation about a possible antiproton excess induced by dark matter annihilations [37]. Understanding secondary production of antimatter in CRs is of fundamental importance, in the context of dark matter searches, in assessing both the signal and background. The positron spectra, the positron fraction $\mathrm{e}^{+} /\left(\mathrm{e}^{-}+\mathrm{e}^{+}\right)$, and the $\bar{p} / p$ ratio from our model will be presented at this conference and reported in a forthcoming work [36]

\section{Connections with Anisotropy and Gamma-Ray Physics}

The study on CR anisotropy in the arrival directions of CRs has received much attention in recent years [34, 38]. The measured $\mathrm{CR}$ anisotropy amplitude in at $\mathrm{TeV}-\mathrm{PeV}$ is found to be nearly constant to $\lesssim 10^{-3}$. From the standard diffusion models, the expected anisotropy exceeds the observational limits by one order of magnitude. To match the data, it is known that a shallower diffusivity is needed, which is in contrast to standard model extrapolations of the $\mathrm{B} / \mathrm{C}$ ratio. In the model considered here, this is not the case. Within the diffusion approximation, our model is able to reconcile the anisotropy amplitude and the $\mathrm{B} / \mathrm{C}$ ratio.

Concerning $\gamma$-ray physics, we note that the Fermi-LAT collaboration has reported a $\gamma$-ray spectral flattening of the spectra in the inner Galaxy in comparison with the outer Galaxy or the halo [39]. These observations find a natural explanation in spatial changes of the CR diffusion properties $[40,17]$.

\section{Conclusions}

Several properties observed in the CR spectrum can found a natural explanation under a diffusive propagation scenario characterized by a spatial change of the CR transport properties in the Galaxy. By change of transport properties, we refer to a different energy dependence of the CR 
diffusion in different region of the Galaxy. An important ingredient is that the diffusion coefficient has to be a non-separable function of energy and space coordinates, in contrast to standard diffusion models that assume homogeneous diffusivity -or space-energy separability- in the whole Galactic halo. A consequence of this scenario is the explanation of the spectral hardening observed in primary CRs, and in particular in the new AMS proton data. Remarkably, this scenario is able to simultaneously accounts for the low-energy steepness of the B/C ratio and for the high-energy requirements of CR anisotropy studies. Important consequences of our scenario deals with the secondary antimatter spectra. More quantitative studies will be presented upon the release of the AMS data on $\mathrm{Li}, \mathrm{B} / \mathrm{C}$ and $\bar{p} / p$.

\section{References}

[1] T. Bell, Braz. J. Phys., 44, 415 (2014);

[2] P. Blasi, Astron. Astrophys. Rev., 21, 70 (2013);

[3] I. A. Grenier, J. H. Black, A. W. Strong, Ann. Rev. Astron. Astroph., 53, 199 (2015);

[4] A. W. Strong, I. V. Moskalenko, V. S. Ptuskin, Ann. Rev. Nucl. Part. Sci., 57, 285-327 (2007);

[5] L. Accardo, et alii, PRL, 113, 121101 (2014);

[6] O. Adriani, Nature, 458, 607-609 (2009);

[7] K. Y. Choi, B. Kyae, C. S. Shin, PRD, 89, 055002 (2014);

[8] K. Blum, B. Katz, E. Waxman, PRL, 111, 211101 (2013);

[9] P. D. Serpico, Astropart. Phys., 39-40, 2-11 (2011);

[10] P. Blasi, PRL, 103, 051104 (2009);

[11] N. Tomassetti, \& F. Donato, ApJ, 803, L15 (2015);

[12] O. Adriani et alii (PAMELA), Science 332, 6025 (2011);

[13] H. S. Yoon, et alii (CREAM), ApJ, 728, 122 (2011);

[14] A. D. Panov et alii (ATIC-2) Bull. Russ. Acad. Sci. 73-5, 602-605 (2009);

[15] M. Aguilar, et alii (AMS), PRL, 114, 171103 (2015);

[16] V. Ptuskin, et alii, ApJ, 763, 47 (2013);

[17] N. Tomassetti, ApJ, 752, L13 (2012);

[18] P. Blasi, E. Amato, P. D. Serpico, PRL 109, 061101 (2012);

[19] A. E. Vladimirov, et alii, ApJ, 752, 68 (2012);

[20] S. Thoudam \& J. Horandel, A\&A, 567, A33 (2014);

[21] G. Giesen, et alii, arXiv:1504.04276 (2015);

[22] R. Kappl, A. Reinert, M. W. Winkler, arXiv:1506.04145 (2015);

[23] R. Cowsik \& T. Madziwa-Nussinov, arXiv:1505.00305 (2015);

[24] C.Evoli, et alii, JCAP, 018 (2008);

[25] C. Jin, Y. Q. Guo, H. B. Hu, arXiv:1504.06903 (2015); 
[26] L. J. Gleeson, \& W. I. Axford, ApJ, 154, 1001 (1968);

[27] O. Adriani, et alii (PAMELA), ApJ, 791, 93 (2014);

[28] H. S. Ahn, et alii (CREAM), 715, 1400-1407 (2010);

[29] A. Obermeier, et alii, ApJ, 752, 69 (2012);

[30] M. Aguilar, et alii, ApJ, 724, 328 (2010);

[31] V. A. Derbina, et alii (RUNJOB), 628, L41 (2005);

[32] C. D. Orth et alii, ApJ, 226, 1147 (1978);

[33] E. Juliusson (HEN), ApJ191, 331 (1974);

[34] M. Pohl \& Eichler, D., ApJ, 766, 4 (2013);

[35] N. Tomassetti \& F. Donato, A\&A, 544, A16 (2012);

[36] N. Tomassetti, in preparation (2015);

[37] C. H. Chem, C. W. Chiang, T. Nomura, PLB, $747495-499$ (2015);

[38] R. Kumar \& D. Eichler ApJ, 785, 129 (2014);

[39] M. Ackermann et alii, ApJ750, 3 (2012);

[40] A. D. Erlykin \& A. Wolfendale, J. Phys. G: NPP,28,2329 (2002);

[41] A. Erlykin \& A. Wolfendale, Astrop.Phy. 42, 70-75 (2013); 